\title{
Fixed Point Methods for the Generalized Stability of Functional Equations in a Single Variable
}

\author{
Liviu Cădariư ${ }^{1}$ and Viorel Radu ${ }^{2}$ \\ ${ }^{1}$ Departamentul de Matematică, Universitatea Politehnica din Timişoara, Piaţa Victoriei no. 2, \\ 300006 Timişoara, Romania \\ ${ }^{2}$ Facultatea de Matematică Şi Informatică, Universitatea de Vest din Timişoara, Bv. Vasile Pârvan 4, \\ 300223 Timişoara, Romania
}

Correspondence should be addressed to Liviu Cădariu, liviu.cadariu@mat.upt.ro

Received 4 October 2007; Accepted 14 December 2007

Recommended by Andrzej Szulkin

We discuss on the generalized Ulam-Hyers stability for functional equations in a single variable, including the nonlinear functional equations, the linear functional equations, and a generalization of functional equation for the square root spiral. The stability results have been obtained by a fixed point method. This method introduces a metrical context and shows that the stability is related to some fixed point of a suitable operator.

Copyright (c) 2008 L. Cădariu and V. Radu. This is an open access article distributed under the Creative Commons Attribution License, which permits unrestricted use, distribution, and reproduction in any medium, provided the original work is properly cited.

\section{Introduction and preliminaries}

The study of functional equations stability originated from a question of Ulam (1940) concerning the stability of group homomorphisms is as follows.

Let $G$ be a group endowed with a metric d. Given $\varepsilon>0$, does there exist a $k>0$ such that for every function $f: G \rightarrow G$ satisfying the inequality

$$
d(f(x \cdot y), f(x) \cdot f(y))<\varepsilon, \quad \forall x, y \in G,
$$

there exists an automorphism $a$ of $G$ with

$$
d(f(x), a(x))<k \varepsilon, \quad \forall x \in G ?
$$

In 1941, Hyers [1] gave an affirmative answer to the question of Ulam for Cauchy equation in Banach spaces.

Let $E_{1}$ and $E_{2}$ be Banach spaces and let $f: E_{1} \rightarrow E_{2}$ be such a mapping that

$$
\|f(x+y)-f(x)-f(y)\| \leq \delta,
$$


for all $x, y \in E_{1}$ and $a \delta>0$, that is, $f$ is $\delta$-additive. Then there exists a unique additive $T: E_{1} \rightarrow E_{2}$, which satisfies

$$
\|f(x)-T(x)\| \leq \delta, \quad \forall x \in E_{1} .
$$

In fact, according to Hyers,

$$
T(x)=\lim _{n \rightarrow \infty} \frac{f\left(2^{n} x\right)}{2^{n}}, \quad \forall x \in E_{1} .
$$

For this reason, one says that the Cauchy equation is stable in the sense of Ulam-Hyers.

In $[2,3]$ as well as in [4-7], the stability problem with unbounded Cauchy differences is considered (see also $[8,9]$ ). Their results include the following two theorems.

Theorem 1.1 (see $[1,2,4,7])$. Suppose that $E$ is a real-normed space, $F$ is a real Banach space, and $f: E \rightarrow F$ is a given function, such that the following condition holds:

$$
\|f(x+y)-f(x)-f(y)\|_{F} \leq \theta\left(\|x\|_{E}^{p}+\|y\|_{E}^{p}\right), \quad \forall x, y \in E,
$$

for some $p \in[0, \infty) \backslash\{1\}$ and $\theta>0$. Then there exists a unique additive function $a: E \rightarrow F$ such that

$$
\|f(x)-a(x)\|_{F} \leq \frac{2 \theta}{\left|2-2^{p}\right|}\|x\|_{E^{\prime}}^{p} \quad \forall x \in E .
$$

Also, if for each $x \in E$ the function $t \rightarrow f(t x)$ from $\mathbb{R}$ to $F$ is continuous for each fixed $x \in E$, then $a$ is linear mapping.

It is worth mentioning that the proofs used the idea conceived by Hyers. Namely, the additive function $a: E \rightarrow F$ is constructed, starting from the given function $f$, by the following formula:

$$
\begin{aligned}
& a(x)=\lim _{n \rightarrow \infty} \frac{1}{2^{n}} f\left(2^{n} x\right), \quad \text { if } p<1, \\
& a(x)=\lim _{n \rightarrow \infty} 2^{n} f\left(\frac{x}{2^{n}}\right), \quad \text { if } p>1 .
\end{aligned}
$$

This method is called the direct method or Hyers' method.

We also mention a result concerning the stability properties with unbounded control conditions invoking products of different powers of norms (see $[5,6,10])$.

Theorem 1.2. Suppose that $E$ is a real-normed space, $F$ is a real Banach space, and $f: E \rightarrow F$ is a given function, such that the following condition holds

$$
\|f(x+y)-f(x)-f(y)\|_{F} \leq \theta\|x\|_{E}^{p} \cdot\|y\|_{E^{\prime}}^{q} \quad \forall x, y \in E,
$$

for some fixed $\theta>0$ and $p, q \in \mathbb{R}$ such that $r=p+q \neq 1$. Then there exists a unique additive function $L: E \rightarrow F$ such that

$$
\|f(x)-L(x)\|_{F} \leq \frac{\theta}{\left|2^{r}-2\right|}\|x\|_{E}^{r}, \quad \forall x \in E .
$$

If in addition $f: E \rightarrow F$ is a mapping such that the transformation $t \rightarrow f(t x)$ is continuous in $t \in \mathbb{R}$, for each fixed $x \in E$, then $L$ is $\mathbb{R}$-linear mapping. 
Generally, whenever the constant $\delta$ in (1.3) is replaced by a control function $(x, y) \rightarrow$ $\delta(x, y)$ with appropriate properties, as in [3], one uses the generic term generalized Ulam-Hyers stability or stability in Ulam-Hyers-Bourgin sense.

In the general case, one uses control conditions of the form

$$
\left\|\Phi_{f}(x, y)\right\| \leq \delta(x, y)
$$

and the stability estimations are of the form

$$
\|f(x)-S(x)\| \leq \varepsilon(x),
$$

where $S$ is a solution, that is, it verifies the functional equation $\Phi_{S}(x, y)=0$, and for $\varepsilon(x)$, explicit formulae are given, which depend on the control $\delta$ as well as on the equation $\Phi_{f}(x, y)$.

We refer the reader to the expository papers [11, 12] or to the books [13-15] (see also the recent articles of Forti $[16,17])$, for supplementary details.

On the other hand, in [18-25], a fixed point method was proposed, by showing that many theorems concerning the stability of Cauchy, Jensen, quadratic, cubic, quartic, and monomial functional equations are consequences of the fixed point alternative. Subsequently, the method has been successfully used, for example, in [26-30]. This method introduces a metrical context and shows that the stability is related to some fixed point of a suitable operator.

The control conditions are responsible for three fundamental facts:

(1) the contraction property of a Schröder-type operator $J$,

(2) the first two approximations, $f$ and $J f$, to be at a finite distance,

(3) they force the fixed point of $J$ to be a solution of the initial equation.

Our main purpose here is to study the generalized stability for some functional equations in a single variable. We prove the generalized Ulam-Hyers stability of the single variable equation

$$
w \circ f \circ \eta=f+h
$$

As an application of our result for (1.8), the stability for the following generalized functional equation of the square root spiral

$$
f\left(p^{-1}(p(x)+k)\right)=f(x)+h(x)
$$

is obtained.

Thereafter, we present the generalized Ulam-Hyers stability of the nonlinear equation

$$
f(x)=F(x, f(\eta(x))) .
$$

The main result is seen to slightly extend the Ulam-Hyers stability previously given in [31, Theorem 2]. As a direct consequence of this result, the generalized Ulam-Hyers stability of the linear equation $f(x)=g(x) \cdot f(\eta(x))+h(x)$ is highlighted. Notice that in all these equations, $f$ is the unknown function and the other ones are given mappings.

Our principal tool is the following fixed point alternative. 
Proposition 1.3 (cf. [32] or [33]). Suppose that a complete generalized metric space (X,d) (i.e., one for which $d$ may assume infinite values) and a strictly contractive mapping $A: X \rightarrow X$ with the Lipschitz constant $L<1$ are given. Then, for a given element $x \in X$, exactly one of the following assertions is true:

$\left(\mathrm{A}_{1}\right) d\left(A^{n} x, A^{n+1} x\right)=+\infty$, for all $n \geq 0$;

$\left(\mathrm{A}_{2}\right)$ there exists $k$ such that $d\left(A^{n} x, A^{n+1} x\right)<+\infty$, for all $n \geq k$.

Actually, if $\left(A_{2}\right)$ holds, then

$\left(\mathrm{A}_{21}\right)$ the sequence $\left(A^{n} x\right)$ is convergent to a fixed point $y^{*}$ of $A$;

$\left(\mathrm{A}_{22}\right) y^{*}$ is the unique fixed point of $A$ in $Y:=\left\{y \in X, d\left(A^{k} x, y\right)<+\infty\right\}$;

$\left(\mathrm{A}_{23}\right) d\left(y, y^{*}\right) \leq(1 /(1-L)) d(y, A y)$, for all $y \in Y$.

\section{A general fixed point method}

Firstly we prove, by the fixed point alternative, a stability result for the single variable equation $w \circ g \circ \eta=g+h$, where

(i) $w$ is a Lipschitz self-mapping (with constant $\ell_{w}$ ) of the Banach space $Y$;

(ii) $\eta$ is a self-mapping of the nonempty set $G$;

(iii) $h: G \rightarrow Y$ is a given function;

(iv) the unknown is a mapping $g: G \rightarrow Y$, that leads to the following.

Theorem 2.1. Suppose that $f: G \rightarrow Y$ satisfies

$$
\|(w \circ f \circ \eta)(x)-f(x)-h(x)\|_{Y} \leq \psi(x), \quad \forall x \in G,
$$

with some given mapping $\psi: G \rightarrow[0, \infty)$. If there exists $L<1$ such that

$$
\ell_{w} \cdot(\psi \circ \eta)(x) \leq L \psi(x), \quad \forall x \in G
$$

then there exists a unique mapping $c: G \rightarrow Y$ which satisfies both the equation

$$
(w \circ c \circ \eta)(x)=c(x)+h(x), \quad \forall x \in G,
$$

and the estimation

$$
\|f(x)-c(x)\|_{Y} \leq \frac{\psi(x)}{1-L}, \quad \forall x \in G
$$

Proof. Let us consider the set $\mathcal{\varepsilon}:=\{g: G \rightarrow Y\}$ and introduce a complete generalized metric on $\varepsilon$ (as usual, $\inf \varnothing=\infty$ ):

$$
d\left(g_{1}, g_{2}\right)=d_{\psi}\left(g_{1}, g_{2}\right)=\inf \left\{K \in \mathbb{R}_{+},\left\|g_{1}(x)-g_{2}(x)\right\|_{Y} \leq K \psi(x), \forall x \in G\right\} .
$$

Now, define the (nonlinear) mapping

$$
J: \mathcal{E} \longrightarrow \mathcal{E}, \quad J g(x):=(w \circ g \circ \eta)(x)-h(x) .
$$


Step 1. Using the hypothesis $\left(\mathbf{H}_{\psi}\right)$ it follows that $J$ is strictly contractive on $\mathcal{\varepsilon}$. Indeed, for any $g_{1}, g_{2} \in \mathcal{E}$ we have

$$
\begin{aligned}
d\left(g_{1}, g_{2}\right) & <K \Longrightarrow\left\|g_{1}(x)-g_{2}(x)\right\|_{Y} \leq K \psi(x), \quad \forall x \in G \\
\left\|J g_{1}(x)-J g_{2}(x)\right\|_{Y} & =\left\|\left(\left(w \circ g_{1} \circ \eta\right)(x)-h(x)\right)-\left(\left(w \circ g_{2} \circ \eta\right)(x)-h(x)\right)\right\|_{Y} \\
& \leq \ell_{w} \cdot\left\|g_{1}(\eta(x))-g_{2}(\eta(x))\right\|_{Y}
\end{aligned}
$$

Therefore

$$
\left\|J g_{1}(x)-J g_{2}(x)\right\|_{Y} \leq \ell_{w} \cdot K \cdot \psi(\eta(x)) \leq K \cdot L \cdot \psi(x), \quad \forall x \in G,
$$

so that $d\left(J g_{1}, J g_{2}\right) \leq L K$, which implies

$$
d\left(J g_{1}, J g_{2}\right) \leq L d\left(g_{1}, g_{2}\right), \quad \forall g_{1}, g_{2} \in \mathcal{\varepsilon} .
$$

This says that $J$ is a strictly contractive self-mapping of $\mathcal{E}$, with the constant $L<1$.

Step 2. $d(f, J f)<\infty$. In fact, using the relation $\left(\mathbf{C}_{\psi}\right)$ it results that $d(f, J f) \leq 1$.

Step 3. We can apply the fixed point alternative and we obtain the existence of a mapping $c: G \rightarrow Y$ such that the following hold.

(i) $c$ is a fixed point of $J$, that is,

$$
(w \circ c \circ \eta)(x)=c(x)+h(x), \quad \forall x \in G .
$$

The mapping $c$ is the unique fixed point of $J$ in the set

$$
\mathcal{F}=\{g \in \mathcal{E}, d(f, g)<\infty\} .
$$

This says that $c$ is the unique mapping verifying both $\left(\mathbf{E}_{w, \eta}\right)$ and $(2.4)$, where

$$
\exists K<\infty \quad \text { such that }\|c(x)-f(x)\|_{Y} \leq K \psi(x), \quad \forall x \in G .
$$

(ii) $d\left(J^{n} f, c\right) \underset{n \rightarrow \infty}{\longrightarrow} 0$, which implies

$$
c(x)=\lim _{n \rightarrow \infty} J^{n} f(x), \quad \forall x \in G,
$$

where

$$
\begin{aligned}
\left(J^{n} f\right)(x) & =\left(w \circ J^{n-1} f \circ \eta\right)(x)-h(x) \\
& =w\left(w\left(J^{n-2} f \circ \eta^{2}\right)(x)-(h \circ \eta)(x)\right)-h(x), \quad \forall x \in G,
\end{aligned}
$$

whence

$J^{n} f=\omega\left(\omega\left(\omega\left(\omega\left(\cdots\left(\omega\left(\omega \circ f \circ \eta^{n}-h \circ \eta^{n-1}\right)-h \circ \eta^{n-2}\right)-\cdots\right)-h \circ \eta^{3}\right)-h \circ \eta^{2}\right)-h \circ \eta\right)-h$.

(iii) Finally, $d(f, c) \leq(1 /(1-L)) d(f, J f)$, which implies the inequality

$$
d(f, c) \leq \frac{1}{1-L}
$$

that is, $\left(\right.$ Est $\left._{\psi}\right)$ is seen to be true. 
Theorem 2.1 extends our recent result in [34], where the generalized stability in UlamHyers sense was obtained for the equation

$$
w \circ g \circ \eta=g
$$

\section{Applications to the generalized equation of the square root spiral}

As a consequence of Theorem 2.1, we obtain a generalized stability result for the equation

$$
f\left(p^{-1}(p(x)+k)\right)=f(x)+h(x), \quad \forall x \in G .
$$

The "unknowns" are functions $f: G \rightarrow Y$ between two vector spaces while $p, h$ are given functions, $p^{-1}$ is the inverse of $p$, and $k \neq 0$ is a fixed constant. The solution of (3.1) and a generalized stability result in Ulam-Hyers sense for the above equation are given in [35], by the direct method.

A vector space $G$ and a Banach space $Y$ will be considered.

Theorem 3.1. Let $k \in G \backslash\{0\}$ and suppose that $p: G \rightarrow G$ is bijective and $h: G \rightarrow Y$ is a given mapping. If $f: G \rightarrow Y$ satisfies

$$
\left\|f\left(p^{-1}(p(x)+k)\right)-f(x)-h(x)\right\|_{Y} \leq \psi(x), \forall x \in G,
$$

with a mapping: $\psi: G \rightarrow[0, \infty)$ for which there exists $L<1$ such that

$$
\psi\left(p^{-1}(p(x)+k)\right)(x) \leq L \psi(x), \quad \forall x \in G,
$$

then there exists a unique mapping $c: G \rightarrow Y$ which satisfies both the equation

$$
c\left(p^{-1}(p(x)+k)\right)=c(x)+h(x), \quad \forall x \in G,
$$

and the estimation

$$
\|f(x)-c(x)\|_{Y} \leq \frac{\psi(x)}{1-L}, \quad \forall x \in G
$$

Moreover,

$$
c(x)=\lim _{n \rightarrow \infty}\left(f\left(p^{-1}(p(x)+n k)\right)-\sum_{i=0}^{n-1} h\left(p^{-1}(p(x)+i k)\right)\right), \quad \forall x \in G .
$$

Proof. We apply Theorem 2.1, with $w: Y \rightarrow Y, \eta: G \rightarrow G, \psi: G \rightarrow[0, \infty)$,

$$
w(x):=x, \quad \eta(x):=p^{-1}(p(x)+k) .
$$

Clearly, $l_{w}=1$ and $J g(x):=g\left(p^{-1}(p(x)+k)\right)-h(x)$.

By using $\left(\mathbf{S}_{\psi}\right)$ and the hypothesis $\left(\mathbf{H}_{\psi, p}\right)$, we immediately see that $\left(\mathbf{C}_{\psi}\right)$ and $\left(\mathbf{H}_{\psi}\right)$ hold.

Since

$$
\eta^{i}(x)=p^{-1}(p(x)+i k), \quad i \in\{1,2, \ldots, n\}
$$


then

$$
\left(J^{n} f\right)(x)=\left(f\left(p^{-1}(p(x)+n k)\right)-\sum_{i=0}^{n-1} h\left(p^{-1}(p(x)+i k)\right)\right)
$$

whence there exists a unique mapping $c: G \rightarrow Y$,

$$
c(x):=\lim _{n \rightarrow \infty}\left(J^{n} f\right)(x), \quad \forall x \in G,
$$

which satisfies the equation $(J c)(x)=c(x)$, that is,

$$
c\left(p^{-1}(p(x)+k)\right)=c(x)+h(x), \quad \forall x \in G,
$$

and the inequality

$$
\|f(x)-c(x)\|_{Y} \leq \frac{\psi(x)}{1-L}, \quad \forall x \in G
$$

A special case of (3.1) is obtained for $k=1, p(x)=x^{n}, n \geq 2$, and $h(x)=\arctan (1 / x)$. It is the so-called " $n$th root spiral equation"

$$
f\left(\sqrt[n]{x^{n}+1}\right)=f(x)+\arctan \frac{1}{x}
$$

As a consequence of Theorem 3.1, we obtain the following generalized stability result for the above equation.

Theorem 3.2. If $f: \mathbb{R}_{+} \rightarrow \mathbb{R}_{+}$satisfies

$$
\left|f\left(\sqrt[n]{x^{n}+1}\right)-f(x)-\arctan \frac{1}{x}\right| \leq \psi(x), \quad \forall x \in \mathbb{R}_{+},
$$

with some fixed mapping $\psi: \mathbb{R}_{+} \rightarrow[0, \infty)$ and there exists $L<1$ such that

$$
\psi\left(\sqrt[n]{x^{n}+1}\right) \leq L \psi(x), \quad \forall x \in \mathbb{R}_{+}
$$

then there exists a unique mapping $c: \mathbb{R}_{+} \rightarrow \mathbb{R}_{+}$,

$$
c(x)=\lim _{m \rightarrow \infty}\left(\sqrt[n]{x^{n}+m}-\sum_{i=0}^{m-1} \arctan \frac{1}{\sqrt[n]{x^{n}+i}}\right), \quad \forall x \in \mathbb{R}_{+},
$$

which satisfies both (3.9) and the estimation

$$
|f(x)-c(x)| \leq \frac{\psi(x)}{1-L}, \quad \forall x \in \mathbb{R}_{+} .
$$

Notice that for $n=2$, Jung and Sahoo [36] proved in 2002 a generalized Ulam-Hyers stability result for the functional equation (3.9), by using the direct method.

If the control mapping $\psi: \mathbb{R}_{+} \rightarrow[0, \infty)$ has the form $\psi(x)=a^{x^{n}}(0<a<1, n \in \mathbb{N})$, a stability result of Aoki-Rassias type for (3.9) is obtained. 
Corollary 3.3. If $f: \mathbb{R}_{+} \rightarrow \mathbb{R}_{+}$satisfies

$$
\left|f\left(\sqrt[n]{x^{n}+1}\right)-f(x)-\arctan \frac{1}{x}\right| \leq a^{x^{n}}, \quad \forall x \in \mathbb{R}_{+}
$$

with some fixed $0<a<1$, then there exists a unique mapping $c: \mathbb{R}_{+} \rightarrow \mathbb{R}_{+}$,

$$
c(x)=\lim _{m \rightarrow \infty}\left(\sqrt[n]{x^{n}+m}-\sum_{i=0}^{m-1} \arctan \frac{1}{\sqrt[n]{x^{n}+i}}\right), \quad \forall x \in \mathbb{R}_{+},
$$

which satisfies both (3.9) and the estimation

$$
|f(x)-c(x)| \leq \frac{a^{x^{n}}}{1-a}, \quad \forall x \in \mathbb{R}_{+}
$$

Proof. We apply Theorem 3.2, by choosing $\psi(x)=a^{x^{n}}(0<a<1, n \in \mathbb{N})$. It is clear that the relation (3.11) holds, with $L=a<1$.

Remark 3.4. A similar result of stability as in Corollary 3.3 can be obtained for a control mapping $\psi: \mathbb{R}_{+} \rightarrow[0, \infty), \psi(x)=\left(1 / a^{x^{n}}\right)(a>1, n \in \mathbb{N})$. The estimation relation (3.16) becomes

$$
|f(x)-c(x)| \leq \frac{a^{1-x^{n}}}{a-1}, \quad \forall x \in \mathbb{R}_{+}
$$

\section{The generalized Ulam-Hyers stability of a nonlinear equation}

The Ulam-Hyers stability for the nonlinear equation

$$
f(x)=F(x, f(\eta(x)))
$$

was discussed by Baker [31]. The "unknowns" are functions $f: G \rightarrow Y$, between two vector spaces. In this section, we will extend the Baker's result and we will obtain the generalized stability in Ulam-Hyers sense for (4.1), by using the fixed point alternative.

Let us consider a nonempty set $G$ and a complete metric space $(Y, d)$.

Theorem 4.1. Let $\eta: G \rightarrow G, g: G \rightarrow \mathbb{R}($ or $\mathbb{C}$ ) and $F: G \times Y \rightarrow Y$. Suppose that

$$
d(F(x, u), F(x, v)) \leq|g(x)| \cdot d(u, v), \quad \forall x \in G, \forall u, v \in Y
$$

If $f: G \rightarrow Y$ satisfies

$$
d(f(x), F(x, f(\eta(x)))) \leq \psi(x), \quad \forall x \in G
$$

with a mapping $\psi: G \rightarrow[0, \infty)$ for which there exists $L<1$ such that

$$
|g(x)|(\psi \circ \eta)(x) \leq L \psi(x), \quad \forall x \in G
$$


then there exists a unique mapping $c: G \rightarrow Y$ which satisfies both the equation

$$
c(x)=F(x, c(\eta(x))), \quad \forall x \in G,
$$

and the estimation

$$
d(f(x), c(x)) \leq \frac{\psi(x)}{1-L}, \quad \forall x \in G
$$

Moreover,

$$
c(x)=\lim _{n \rightarrow \infty} F\left(x, F\left(F\left(\eta(x), \ldots, F\left(\eta(x),\left(f \circ \eta^{n}\right)(\eta(x))\right)\right)\right)\right), \quad \forall x \in G
$$

Proof. We use the same method as in the proof of Theorem 2.1, namely, the fixed point alternative. Let us consider the set $\varepsilon:=\{h: G \rightarrow Y\}$ and introduce a complete generalized metric on $\varepsilon$ (as usual, $\inf \varnothing=\infty$ ):

$$
\rho\left(h_{1}, h_{2}\right)=\inf \left\{K \in \mathbb{R}_{+}, d\left(h_{1}(x), h_{2}(x)\right) \leq K \psi(x), \forall x \in G\right\}
$$

Now, define the mapping

$$
J: \varepsilon \longrightarrow \mathcal{\varepsilon}, \quad J h(x):=F(x, h(\eta(x)))
$$

Step 1. Using the hypothesis in (4.2) and (4.4) it follows that $J$ is strictly contractive on $\varepsilon$. Indeed, for any $h_{1}, h_{2} \in \mathcal{E}$ we have

$$
\begin{aligned}
\rho\left(h_{1}, h_{2}\right) & <K \Longrightarrow d\left(h_{1}(x), h_{2}(x)\right) \leq K \psi(x), \quad \forall x \in G, \\
d\left(J h_{1}(x), J h_{2}(x)\right) & =d\left(F\left(x, h_{1}(\eta(x))\right), F\left(x, h_{2}(\eta(x))\right)\right) \\
& \leq|g(x)| \cdot d\left(h_{1}(\eta(x)), h_{2}(\eta(x))\right) \\
& \leq K \cdot|g(x)| \cdot \psi(\eta(x)) .
\end{aligned}
$$

Therefore

$$
d\left(J h_{1}(x), J h_{2}(x)\right) \leq K \cdot|g(x)| \cdot \psi(\eta(x)) \leq K \cdot L \cdot \psi(x), \quad \forall x \in G
$$

so that $\rho\left(J h_{1}, J h_{2}\right) \leq L K$, which implies

$$
\rho\left(J h_{1}, J h_{2}\right) \leq L \rho\left(h_{1}, h_{2}\right), \quad \forall h_{1}, h_{2} \in \mathcal{\varepsilon}
$$

This says that $J$ is a strictly contractive self-mapping of $\mathcal{E}$, with the constant $L<1$.

Step 2. Obviously, $\rho(f, J f)<\infty$. In fact, the relation (4.3) implies $\rho(f, J f) \leq 1$. 
Step 3. We can apply the fixed point alternative (see Proposition 1.3), and we obtain the existence of a mapping $c: G \rightarrow Y$ such that the following hold.

(i) $c$ is a fixed point of $J$, that is,

$$
c(x)=F(x, c(\eta(x))), \quad \forall x \in G .
$$

The mapping $c$ is the unique fixed point of $J$ in the set

$$
\mathcal{F}=\{h \in \mathcal{E}, \rho(f, h)<\infty\} .
$$

This says that $c$ is the unique mapping verifying both (4.13) and (4.15) where

$$
\exists K<\infty \text { such that } d(c(x), f(x)) \leq K \psi(x), \quad \forall x \in G .
$$

(ii) $\rho\left(J^{n} f, c\right) \underset{n \rightarrow \infty}{\longrightarrow} 0$, which implies

$$
c(x)=\lim _{n \rightarrow \infty} J^{n} f(x), \quad \forall x \in G,
$$

where

$$
\begin{aligned}
\left(J^{n} f\right)(x) & =F\left(x,\left(J^{n-1} f\right)(\eta(x))\right) \\
& =F\left(x, F\left(\eta(x),\left(J^{n-2} f\right)(\eta(x))\right)\right), \quad \forall x \in G,
\end{aligned}
$$

hence

$$
\left(J^{n} f\right)(x)=F\left(x, F\left(F\left(\eta(x), \ldots F\left(\eta(x),\left(f \circ \eta^{n}\right)(\eta(x))\right)\right)\right) .\right.
$$

(iii) $\rho(f, c) \leq(1 /(1-L)) \rho(f, J f)$, which implies the inequality

$$
\rho(f, c) \leq \frac{1}{1-L}
$$

that is, (4.6) holds.

As a direct consequence of Theorem 4.1, the following Ulam-Hyers stability result (cf. [31, Theorem 2] or [37, Theorem 13]) for the nonlinear equation (4.1) is obtained.

Corollary 4.2. Let $G$ be a nonempty set and let $(Y, d)$ be a complete metric space. Let $\eta: G \rightarrow G$, $F: G \times Y \rightarrow Y$, and $0 \leq L<1$. Suppose that

$$
d(F(x, u), F(x, v)) \leq L \cdot d(u, v), \quad \forall x \in G, \forall u, v \in Y .
$$

If $f: G \rightarrow Y$ satisfies

$$
d(f(x), F(x, f(\eta(x)))) \leq \delta, \quad \forall x \in G,
$$

with a fixed constant $\delta>0$, then there exists a unique mapping $c: G \rightarrow Y$ which satisfies both the equation

$$
c(x)=F(x, c(\eta(x))), \quad \forall x \in G,
$$

and the estimation

$$
d(f(x), c(x)) \leq \frac{\delta}{1-L}, \quad \forall x \in G
$$


Moreover,

$$
c(x)=\lim _{n \rightarrow \infty} F\left(x, F\left(F\left(\eta(x), \ldots, F\left(\eta(x),\left(f \circ \eta^{n}\right)(\eta(x))\right)\right)\right)\right), \quad \forall x \in G .
$$

Proof. It follows by Theorem 4.1 , by choosing $\psi(x)=\delta>0$.

Example 4.3. If in (4.1) we consider $F: \mathbb{R} \times[1, \infty) \rightarrow[1, \infty)$,

$$
F(x, u)= \begin{cases}u^{1 / p}, & \text { if } p>1, \\ u^{p}, & \text { if } 0<p<1,\end{cases}
$$

we obtain the equation of Böttcher:

$$
(f(\eta(x)))^{1 / p}=f(x), \quad p>1, \quad \text { or } \quad f(\eta(x))^{p}=f(x), \quad 0<p<1 .
$$

Agarwal et al. proved in [37, Theorem 14] that the above equations are stable in Ulam-Hyers sense, by using the result of Baker [31], with

$$
L= \begin{cases}\frac{1}{p^{\prime}} & \text { if } p>1 \\ p, & \text { if } 0<p<1\end{cases}
$$

It is worth noticing that our formula (4.24) gives the solutions $c$ of (4.26):

$$
c(x)= \begin{cases}\lim _{n \rightarrow \infty}\left(f\left(\eta^{n}(x)\right)\right)^{1 / p^{n}}, & \text { if } p>1, \forall x \in \mathbb{R}, \\ \lim _{n \rightarrow \infty}\left(f\left(\eta^{n}(x)\right)\right)^{p^{n}}, & \text { if } 0<p<1, \forall x \in \mathbb{R} .\end{cases}
$$

\section{The generalized Ulam-Hyers stability of a linear functional equation}

In this section, we emphasize the importance of Theorem 4.1. In fact, if

$$
F(x, f(\eta(x)))=g(x) \cdot f(\eta(x))+h(x)
$$

equation (4.1) becomes

$$
f(x)=g(x) \cdot f(\eta(x))+h(x)
$$

where $g, \eta, h$ are given mappings and $f$ is unknown function. The above equation is called linear functional equation and was intensively investigated by Kuczma et al. [38]. They obtained some results concerning monotonic solutions, regular solutions, and convex solutions of (5.2).

In what follows we prove a generalized Ulam-Hyers stability result for (5.2), as a particular case of Theorem 4.1 (see also [39]). We also show that the generalized stability of (3.1) can be obtained as consequence of the following theorem. 
Theorem 5.1. Consider $G$ a nonempty set and $Y$ a real (or complex) Banach space. Suppose that $\eta$ : $G \rightarrow G, g: G \rightarrow \mathbb{R}$ (or $\mathbb{C}$ ). If $f: G \rightarrow Y$ satisfies

$$
\|f(x)-g(x) f(\eta(x))-h(x)\|_{Y} \leq \psi(x), \quad \forall x \in G,
$$

with some fixed mapping $\psi: G \rightarrow[0, \infty)$ and there exists $L<1$ such that

$$
|g(x)|(\psi \circ \eta)(x) \leq L \psi(x), \quad \forall x \in G
$$

then there exists a unique mapping $c: G \rightarrow Y$,

$$
c(x)=h(x)+\lim _{n \rightarrow \infty}\left(f\left(\eta^{n}(x)\right) \cdot \prod_{i=0}^{n-1} g\left(\eta^{i}(x)\right)+\sum_{j=0}^{n-2}\left(h\left(\eta^{j+1}(x)\right) \cdot \prod_{i=0}^{j} g\left(\eta^{i}(x)\right)\right)\right)
$$

for all $x \in G$, which satisfies both the equation

$$
c(x)=g(x) \cdot c(\eta(x))+h(x), \quad \forall x \in G,
$$

and the estimation

$$
\|f(x)-c(x)\|_{Y} \leq \frac{\psi(x)}{1-L}, \quad \forall x \in G
$$

Proof. We consider in Theorem 4.1 the metric $d$ on $Y$, given by $d(u, v)=\|u-v\|_{Y}$ and the function

$$
F(x, f(\eta(x))):=g(x) f(\eta(x))+h(x), \quad \forall x \in G,
$$

with $g, \eta, h$ as in hypothesis of Theorem 5.1. The relation (4.2) holds with equality. Applying Theorem 4.1, there exists a unique mapping $c$ which satisfies (5.2) and the estimation (5.7). Moreover,

$$
c(x)=\lim _{n \rightarrow \infty} J^{n} f(x), \quad \forall x \in G,
$$

where

$$
\begin{aligned}
\left(J^{n} f\right)(x) & =g(x) \cdot\left(J^{n-1} f\right)(\eta(x))+h(x) \\
& =g(x) \cdot g(\eta(x)) \cdot\left(J^{n-2} f\right)\left(\eta^{2}(x)\right)+g(x) \cdot h(\eta(x))+h(x), \quad \forall x \in G,
\end{aligned}
$$

whence, for all $x \in G$,

$$
J^{n} f(x):=h(x)+f\left(\eta^{n}(x)\right) \cdot \prod_{i=0}^{n-1} g\left(\eta^{i}(x)\right)+\sum_{j=0}^{n-2}\left(h\left(\eta^{j+1}(x)\right) \cdot \prod_{i=0}^{j} g\left(\eta^{i}(x)\right)\right) .
$$

If $\psi(x)=\delta>0$ in the above Theorem 5.1, then we will obtain the Ulam-Hyers stability result of Baker [31, Theorem 3] (see also [37, Theorem 7] for the linear equation (5.2)). 
Corollary 5.2. Consider a nonempty set $G$ and a real (or complex) Banach space $Y$. Suppose that $\eta: G \rightarrow G, g: G \rightarrow \mathbb{R}($ or $\mathbb{C}$ ), and $h: G \rightarrow Y$ are given. If $f: G \rightarrow Y$ satisfies

$$
\|f(x)-g(x) f(\eta(x))-h(x)\|_{Y} \leq \delta, \quad \forall x \in G,
$$

with a fixed constant $\delta>0$ and there exists $L<1$ such that

$$
|g(x)| \leq L, \quad \forall x \in G
$$

then there exists a unique mapping $c: G \rightarrow Y$,

$$
c(x)=h(x)+\lim _{n \rightarrow \infty}\left(f\left(\eta^{n}(x)\right) \cdot \prod_{i=0}^{n-1} g\left(\eta^{i}(x)\right)+\sum_{j=0}^{n-2}\left(h\left(\eta^{j+1}(x)\right) \cdot \prod_{i=0}^{j} g\left(\eta^{i}(x)\right)\right)\right),
$$

for all $x \in G$, which satisfies both the equation

$$
c(x)=g(x) \cdot c(\eta(x))+h(x), \quad \forall x \in G
$$

and the estimation

$$
\|f(x)-c(x)\|_{Y} \leq \frac{\delta}{1-L}, \quad \forall x \in G
$$

Remark 5.3. It is easy to see that (3.1) is a particular case of (5.2). To prove this, it is sufficient to consider in (5.2) $g \equiv 1, h:=-h_{1}, \eta(x):=p^{-1}(p(x)+k), \forall x \in G$, with $p$ bijective on $G$ and $k \neq 0$ a fixed constant. By using the above notations, Theorem 3.1 can be obtained as a consequence of Theorem 5.1, with

$$
\begin{aligned}
c(x) & =h(x)+\lim _{n \rightarrow \infty}\left(f\left(\eta^{n}(x)\right) \cdot \prod_{i=0}^{n-1} g\left(\eta^{i}(x)\right)+\sum_{j=0}^{n-2}\left(h\left(\eta^{j+1}(x)\right) \cdot \prod_{i=0}^{j} g\left(\eta^{i}(x)\right)\right)\right) \\
& =-h_{1}(x)+\lim _{n \rightarrow \infty}\left(f\left(p\left(p^{-1}(x)+n k\right)\right)-\sum_{i=1}^{n-1} h_{1}\left(p\left(p^{-1}(x)+i k\right)\right)\right) \\
& =\lim _{n \rightarrow \infty}\left(f\left(p^{-1}(p(x)+n k)\right)-\sum_{i=0}^{n-1} h_{1}\left(p^{-1}(p(x)+i k)\right)\right), \quad \forall x \in G .
\end{aligned}
$$

\section{Acknowledgment}

The authors would like to thank the referees and the editors for their help and suggestions in improving this paper.

\section{References}

[1] D. H. Hyers, "On the stability of the linear functional equation," Proceedings of the National Academy of Sciences of the United States of America, vol. 27, no. 4, pp. 222-224, 1941.

[2] T. Aoki, "On the stability of the linear transformation in Banach spaces," Journal of the Mathematical Society of Japan, vol. 2, pp. 64-66, 1950. 
[3] D. G. Bourgin, "Classes of transformations and bordering transformations," Bulletin of the American Mathematical Society, vol. 57, pp. 223-237, 1951.

[4] Z. Gajda, "On stability of additive mappings," International Journal of Mathematics and Mathematical Sciences, vol. 14, no. 3, pp. 431-434, 1991.

[5] J. M. Rassias, "On approximation of approximately linear mappings by linear mappings," Journal of Functional Analysis, vol. 46, no. 1, pp. 126-130, 1982.

[6] J. M. Rassias, "Solution of a problem of Ulam," Journal of Approximation Theory, vol. 57, no. 3, pp. 268-273, 1989.

[7] T. M. Rassias, "On the stability of the linear mapping in Banach spaces," Proceedings of the American Mathematical Society, vol. 72, no. 2, pp. 297-300, 1978.

[8] G. L. Forti, "An existence and stability theorem for a class of functional equations," Stochastica, vol. 4, no. 1 , pp. 23-30, 1980.

[9] P. Găvruţa, "A generalization of the Hyers-Ulam-Rassias stability of approximately additive mappings," Journal of Mathematical Analysis and Applications, vol. 184, no. 3, pp. 431-436, 1994.

[10] J. M. Rassias, "On the stability of the Euler-Lagrange functional equation," Comptes Rendus de l' Académie Bulgare des Sciences, vol. 45, no. 6, pp. 17-20, 1992.

[11] G. L. Forti, "Hyers-Ulam stability of functional equations in several variables," Aequationes Mathematicae, vol. 50, no. 1-2, pp. 143-190, 1995.

[12] T. M. Rassias, "On the stability of functional equations and a problem of Ulam," Acta Applicandae Mathematicae, vol. 62, no. 1, pp. 23-130, 2000.

[13] Z. Daroczy and Z. Pales, Eds., Functional Equations—Results and Advances, vol. 3 of Advances in Mathematics, Kluwer Academic Publishers, Dordrecht, The Netherlands, 2002.

[14] D. H. Hyers, G. Isac, and T. M. Rassias, Stability of functional equations in several variables, vol. 34 of Progress in Nonlinear Differential Equations and Their Applications, Birkhäuser, Basel, Switzerland, 1998.

[15] S.-M. Jung, Hyers-Ulam-Rassias Stability of Functional Equations in Mathematical Analysis, Hadronic Press, Palm Harbor, Fla, USA, 2001.

[16] G. L. Forti, "Comments on the core of the direct method for proving Hyers-Ulam stability of functional equations," Journal of Mathematical Analysis and Applications, vol. 295, no. 1, pp. 127-133, 2004.

[17] G. L. Forti, "Elementary remarks on Ulam-Hyers stability of linear functional equations," Journal of Mathematical Analysis and Applications, vol. 328, no. 1, pp. 109-118, 2007.

[18] L. Cădariu and V. Radu, "Fixed points and the stability of Jensen's functional equation," Fixed Point Theory, vol. 4, no. 1, Article 4, p. 7, 2003.

[19] L. Cădariu and V. Radu, "Fixed points and the stability of quadratic functional equations," Analele Universităţii de Vest din Timişoara, vol. 41, no. 1, pp. 25-48, 2003.

[20] L. Cădariu and V. Radu, "On the stability of the Cauchy functional equation: a fixed point approach," in Iteration Theory (ECIT '02), J. S. Ramos, D. Gronau, C. Mira, L. Reich, and A. N. Sharkovsky, Eds., vol. 346 of Grazer Mathematische Berichte, pp. 43-52, Karl-Franzens-Univ. Graz, Graz, Austria, 2004.

[21] L. Cădariu and V. Radu, "A Hyers-Ulam-Rassias stability theorem for a quartic functional equation," Automation Computers and Applied Mathematics, vol. 13, no. 1, pp. 31-39, 2004.

[22] L. Cădariu and V. Radu, "Fixed points in generalized metric spaces and the stability of a cubic functional equation," in Fixed Point Theory and Applications, Y. J. Cho, J. K. Kim, and S. M. Kang, Eds., vol. 7, pp. 53-68, Nova Science Publishers, Hauppauge, NY, USA, 2007.

[23] L. Cădariu and V. Radu, "The alternative of fixed point and stability results for functional equations," International Journal of Applied Mathematics \& Statistics, vol. 7, no. Fe07, pp. 40-58, 2007.

[24] L. Cădariu and V. Radu, "Remarks on the stability of monomial functional equations," Fixed Point Theory, vol. 8, no. 2, pp. 201-218, 2007.

[25] V. Radu, "The fixed point alternative and the stability of functional equations," Fixed Point Theory, vol. 4, no. 1, pp. 91-96, 2003.

[26] S.-M. Jung and T.-S. Kim, "A fixed point approach to the stability of the cubic functional equation," Boletín de la Sociedad Matemática Mexicana. Tercera Serie, vol. 12, no. 1, pp. 51-57, 2006.

[27] S.-M. Jung, "A fixed point approach to the stability of isometries," Journal of Mathematical Analysis and Applications, vol. 329, no. 2, pp. 879-890, 2007.

[28] S.-M. Jung, "A fixed point approach to the stability of a Volterra integral equation," Fixed Point Theory and Applications, vol. 2007, Article ID 57064, 9, 2007.

[29] M. Mirzavaziri and M. S. Moslehian, "A fixed point approach to stability of a quadratic equation," Bulletin of the Brazilian Mathematical Society, vol. 37, no. 3, pp. 361-376, 2006. 
[30] J. M. Rassias, "Alternative contraction principle and Ulam stability problem," Mathematical Sciences Research Journal, vol. 9, no. 7, pp. 190-199, 2005.

[31] J. A. Baker, "The stability of certain functional equations," Proceedings of the American Mathematical Society, vol. 112, no. 3, pp. 729-732, 1991.

[32] J. B. Diaz and B. Margolis, "A fixed point theorem of the alternative, for contractions on a generalized complete metric space," Bulletin of the American Mathematical Society, vol. 74, pp. 305-309, 1968.

[33] I. A. Rus, Principii şi Aplicații ale Teoriei Punctului Fix, Editura Dacia, Cluj-Napoca, Romania, 1979.

[34] L. Cădariu and V. Radu, "The fixed points method for the stability of some functional equations," Carpathian Journal of Mathematics, vol. 23, no. 1-2, pp. 63-72, 2007.

[35] Z. Wang, X. Chen, and B. Xu, "Generalization of functional equation for the square root spiral," Applied Mathematics and Computation, vol. 182, no. 2, pp. 1355-1360, 2006.

[36] S.-M. Jung and P. K. Sahoo, "Stability of a functional equation for square root spirals," Applied Mathematics Letters, vol. 15, no. 4, pp. 435-438, 2002.

[37] R. P. Agarwal, B. Xu, and W. Zhang, "Stability of functional equations in single variable," Journal of Mathematical Analysis and Applications, vol. 288, no. 2, pp. 852-869, 2003.

[38] M. Kuczma, B. Choczewski, and R. Ger, Iterative functional equations, vol. 32 of Encyclopedia of Mathematics and Its Applications, Cambridge University Press, Cambridge, UK, 1990.

[39] T. Trif, "On the stability of a general gamma-type functional equation," Publicationes Mathematicae Debrecen, vol. 60, no. 1-2, pp. 47-61, 2002. 